\title{
Electron beam cooling by laser
}

J.Urakawa, K.Kubo, N.Terunuma, T.Taniguchi, Y.Yamazaki, K.Hirano ${ }^{1}$, M.Nomura ${ }^{1}$,

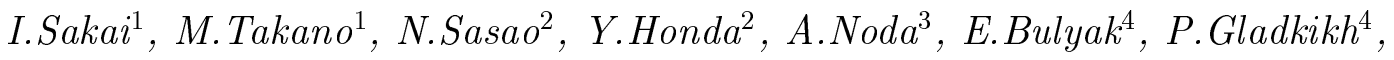

$$
\text { A.Mytsykov }{ }^{4} \text {, A.Zelinsky }{ }^{4}, \text { F.Zimmermann }{ }^{5}
$$

High Energy Accelerator Research Organization(KEK), 1-1 Oho, Tsukuba-shi, Ibaraki,

\author{
Japan

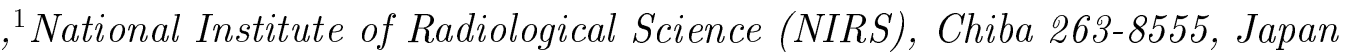 \\ ${ }^{2}$ Department of Physics Kyoto University, Kyoto 606-8502, Japan \\ ${ }^{3}$ Institute for Chemical Research, Kyoto University, Gokanosho, Uji, Kyoto, 611-0011, \\ Japan \\ ${ }^{4}$ NSC KIPT, Kharkov, Ukraine \\ ${ }^{5}$ European Organization for Nuclear Research (CERN), Geneva, Switzerland \\ e-mail: Junji.Urakawa@kek.jp
}

\begin{abstract}
In 1997, Z.Huang and R.Ruth proposed a compact laser-electron storage ring (LESR) for electron beam cooling or x-ray generation. Because the laser-wire monitor in the ATF storage ring has worked well and demonstrated the achievement of the world's smallest transverse emittance for a circulating electron beam, we have started the design of a small storage ring with about $10 \mathrm{~m}$ circumference and the development of basic technologies for the LESR. In this paper, we describe the design and experimental results of pulse stacking in a 42-cm long optical cavity. Since our primary purpose is demonstrating
\end{abstract}


the proof-of-principle of the LESR, we will then discuss the future experimental plan at the KEK-ATF for the generation of high average-brilliance gamma-rays. PACS codes: 29.20.Dh;07.85.Fv;29.27.Eg

Keywords: Emittance; Storage ring; Intra-beam scattering; Synchrotron radiation; Brightness

\section{Introduction}

Recent proposals[1,2] concerning the radiative laser cooling of electron beams for the production of intense beams of quasi-monoenergetic X-rays have attracted the attention of the accelerator community. If we assume an enhancement factor of 20000 in the laser power and a $50 \mu \mathrm{m}$ rms laser-beam size at the beam-laser interaction point (IP), which can be achieved by using a high-finesse optical cavity, the peak power exceeds $1 \mathrm{TW} / \mathrm{cm}^{2}$ in the case of a 7 ps FWHM pulsed laser. Mode-locked lasers with these parameters are commercially available (for example $N d: Y V O_{4}$ ). This power lies well within the linear Compton scattering region.

We have designed an electron storage ring for medical applications. Its circumference is $13.44 \mathrm{~m}$, the beam energy ranges from 40 to $60 \mathrm{MeV}$, and the energy acceptance amounts to $\pm 3 \%$. In parallel, a $42 \mathrm{~cm}$ long optical cavity was constructed which can provide a beam-laser interaction at a 10 degree crossing angle for medical applications. The corresponding X-ray yield is about $10 \%$ of that for a head-on collision. A longer optical cavity $(1.68 \mathrm{~m})$ and a multi-laser pulse in the cavity would increase the X-ray flux.

We also intend to conduct a proof-of-principle experiment for the radiative laser cool- 
ing at the KEK-ATF. Assuming the same 10 degree crossing as for the LESR design, electrons stored in the KEK-ATF [3] damping ring would quickly be lost due to the repeated interaction with the laser and top-up injection would be necessary. So, at the KEK-ATF, we will mainly study the timing jitter and alignment issues. From the experimental results we can then extrapolate to the high average-brilliance $\gamma$-ray generation.

The LESR uses an intense laser pulse stored in a high-finesse resonator, which repetitively interacts with the circulating electron beam. The rapid damping caused by the laser-electron interaction counterbalances the intrabeam scattering effect, thus allowing electron beams of relatively low energy to be cooled or stabilized in the storage ring, down to extremely low transverse emittances.

In the following sections we describe the laser-wire technologies for inverse Compton scattering which have been developed at KEK-ATF: first the proof-of-principle experiment on laser pulse stacking, next the planned stability study on Compton scattering at the KEK-ATF, and then the experimental plan for radiative laser cooling itself. Finally, a discussion and summary are given.

\section{Laser-wire technologies for inverse Compton scattering}

We have developed a laser-wire beam profile monitor for measuring the electron-beam emittance at the KEK-ATF. This monitor is based on the inverse Compton scattering with a laser light target. A thin and intense laser target is produced by exciting a FabryPerot optical cavity with a CW laser. In the process of this monitor development, the following technologies have been established [4]: 
- A relative positioning of the mirrors with a precision of $0.1 \mathrm{~nm}(\mathrm{rms})$ was achieved, using a feedback system based on piezoelectric transducers (PZT). This precision corresponds to a width control of the laser-wire size within $5 \mu \mathrm{m}$ rms.

- The mode matching technique, required for maintaining a power enhancement factor above 1000, was precisely demonstrated. The ultimate enhancement factor depends on the reflectivity of the mirrors.

- We found that the electron rms beam orbit jitter in the storage ring is less than $1 \mu \mathrm{m}$ rms.

- The laser-wire position was stabilized to within $0.1 \mu \mathrm{m}$ rms.

\section{Proof-of-principle experiment on laser-pulse stacking}

We have started the proof-of-principle experiment on short pulsed laser stacking in a Fabry-Perot optical cavity. We selected a commercially available mode-locked laser (High Q Laser IC-6000 ps VAN357) and a 42-cm optical cavity for this experiment. For an easy and quick study, we chose a very simple set-up, consisting of the modelocked laser, the optical system, $90 \%$ reflective mirrors and several detectors, as shown in Fig. 1. The frequency of the mode-locker was modulated for measuring the cavity finesse during the pulse stacking. This means that the central frequency of the longitudinal mode from the mode-locked laser oscillator is shifted. If the central frequency is changed by about $500 \mathrm{~Hz}$ which corresponds half a wavelength of $1064 \mathrm{~nm}$, the optical cavity for the pulse stacking oscillates between reflection and accumulation of the laser pulse. Table 1 shows the results of a simple measurement, indicating a good agreement with 
the calculation for the employed $90 \%$ reflective mirrors. The latter are also commercially available and of spherical type (radius $0.5 \mathrm{~m}$ ). The $39 \%$ discrepancy between calculation and measurement in the transmission arises from the absorption by the mirror material, errors in the surface curvature of the reflective coating, for which a precision at the subnanometer level is required, and an insufficient optical mode matching. The table also lists the values calculated for higher-quality mirrors, whose employment would benefit from the other technologies developed for the laser-wire monitor. In the near future, we will add a stepping motor and piezoelectric actuators (PZT) to control the length of the optical cavity as is done for the mode-locked laser oscillator. Figure 2 shows a conceptual diagram for the proof-of-principle experiment on radiative laser cooling. The laser oscillator, the pulse-stacking system and the beam of the storage ring are synchronized by a single signal generator with a relative frequency stability of $10^{-13}$. This synthesizer and also mirrors with $99.5 \%, 99.9 \%, 99.99 \%$ and $99.999 \%$ reflectivity are available from various companies. The high-reflection mirrors exhibit an ultralow loss of a only few ppm from scattering and absorption [5].

\section{Stability study on Compton scattering at KEK-ATF}

The laser-wire beam profile monitor at the KEK-ATF is working well and it measures the beam profile of each bunch in multi-bunch operation. If this monitor is replaced by the optical cavity for the laser-pulse stacking, we can check the stability of the waist size of the pulsed laser beam using the low-emittance electron beam as a probe. The scheme of Fig. 2 promises a precise synchronization within 0.1 psec. However, we have to confirm that this number can be achieved under practical conditions and demonstrate 
the generation of $10^{13} \gamma$-rays per second.

\section{Experimental plan of radiative laser cooling}

In nonconservative systems, a steady-state particle distribution can be established due to a balance of cooling and heating processes. In an electron storage ring, the only cooling process is the radiation damping. We here consider an electron beam with natural emittance $\epsilon_{n}$ due to the synchrotron radiation power from bending magnets $p_{n}$. If, in addition, a radiation damping by laser-Compton scattering is present, characterized by the partial emittance $\epsilon_{l}$ and the radiation power $p_{l}$, the steady-state emittance without the intra-beam scattering effect becomes

$$
\epsilon=\frac{p_{n} \epsilon_{n}+p_{l} \epsilon_{l}}{p_{n}+p_{l}}
$$

where we treated the bending-magnet synchrotron radiation and the laser-beam interaction as statistically independent.

For a ring in which the laser-beam interaction point is located in a dispersion-free section, the horizontal partial Compton transverse emittance $\epsilon_{l}$ is much smaller than the partial emittance from synchrotron radiation:

$$
\epsilon_{l}=\frac{3 \beta_{I P}}{20} \frac{E_{l a s}}{\gamma E_{0}}(1+\cos \varphi) \ll \epsilon_{n},
$$

where $\gamma$ is the electron Lorents factor, $\beta_{I P}$ the horizontal beta function at the IP, $\varphi$ the collision angle, $E_{\text {las }}$ the energy of a laser photon, and $E_{0}$ the electron rest energy. These considerations motivate a novel type of storage rings which is optimized for the demonstration of the radiative laser-cooling effect. 


\section{5-1. Design of small storage ring}

A small storage ring has therefore been designed for the proof-of-principle experiment on the radiative laser cooling and high brightness X-ray generation for medical application. Figure 3 shows the ring layout, which fits on a large table. We arrange for one IP in a dispersion-free section, where we will install a 1.68-m long optical resonator, which stores 4 laser pulses of $0.5 \mathrm{~mJ}$ energy each. The rms waist size of the laser flash is $50 \mu \mathrm{m}$. In the ring, 16 bunches of $2 \mathrm{nC}$ each are circulating, uniformly distributed around the circumference (every other RF bucket is left empty).

\section{5-2. Energy acceptance}

We calculated the energy acceptance using dedicated particle tracking codes which include the intra-beam scattering effect (IBS), the laser radiative cooling and the synchrotronradiation process. From the simulation results, we infer the long-term transverse stability border of particle motion with a maximum momentum deviation of $\pm 3.0 \%$, i.e., the offmomentum dynamic aperture.

Due to the Compton interaction, the energy spread increases. The partial Compton energy spread is:

$$
\delta^{2}=\frac{7}{10} \gamma \frac{E_{l a s}}{E_{0}} \frac{1+\cos \varphi}{2}
$$

The steady state energy spread due to both synchrotron and Compton fluctuations can be derived from Eq.(1), where emittances should be replaced by the squared energy spreads without IBS. Thus, the total energy spread in the steady state depends on the intensity of the Compton interaction.

\section{Discussion and summary}


In the case of $3.0 \times 10^{9}$ electrons per bunch and $10 \%$ coupling, the steady-state longitudinal emittance is $0.252 \% \times 10.20 \mathrm{~mm}=2.6 \times 10^{-5} \mathrm{~m}$ without the laser-Compton scattering, estimating the effect of intra-beam scattering by the BM model [6]. For an electron bunch of $2 \mathrm{nC}$ (about $1.3 \times 10^{10}$ electrons) and again $10 \%$ coupling, it approaches $1.0 \times 10^{-4} \mathrm{~m}$, provided the RF accelerating voltage is sufficient $(0.4 \mathrm{MV})$. The storage ring will be equipped with a $714 \mathrm{MHz}$ RF system, which can accommodate a beam of $11.67 \mathrm{~mm}$ rms bunch length and $1.67 \%$ rms energy spread, as expected to result from the quantum fluctuation of the Compton scattering[7]. Since the longitudinal direction is heated by both the laser-Compton scattering and the intra-beam scattering, a momentum acceptance of $3.0 \%$ is necessary to alleviate chromatic effects at the laser-beam interaction point and to ensure an adequate dynamic aperture. In a ring with the target momentum acceptance, we can circulate the electron beam together with an extremely high-power laser pulse, e.g., $5 \mathrm{~mJ} /$ pulse, assuming that the ideal theory of Compton scattering applies [8].

We have roughly estimated the yield of X-rays scattered along the electron trajectory within the solid angle of $1 / \gamma$. It is $10^{14}$ photons per second and applicable for digital differential angiography.

\section{Acknowledgements}

We would like to thank all members of the KEK-ATF group for their helpful support and discussion. This research was partially supported by the budget for the Advanced Compact Accelerator Project of the National Institute of Radiological Sciences 


\section{References}

[1] Zh. Huang and R.D. Ruth, Phys. Rev. Lett., Vol.80, No.5, 1998, p.976.

[2] V. Telnov, SLAC-PUB-NSF-ITP-96-142, 1996.

[3] Edited by F. Hinode et al., KEK Internal 95-4, 1995.

[4] Y. Honda et al., to be submitted to Nucl. Instr. and Meth.

[5] G. Rempe et al., Optics Letters, Vol.17, No.5, 1992, p.363.

[6] J.D.Bjorken and S.K.Mtingwa, Particle Accelerator, Vol.13, p.115(1983).

[7] I. Karnaukhov, A. Zelinsky, and Yu. Telegin, Nucl. Instr. and Meth. in Physics Research A 470, 2001, p.23.

[8] E. Bulyak et al., Nucl. Instr. and Meth. in Physics Research A 487, 2002, p.241. 
Figure 1 Schematic diagram of the experimental set-up for laser-pulse stacking.

Figure 2 Conceptual diagram for the proof-of-principle experiment on radiative laser cooling. The $99.99 \%$ reflective mirror which has surface roughness less than $0.1 \mathrm{~nm}$ is available. Phase locking accuracy is still open-question.

Figure 3 Ring layout of C3X (Compact Clean Compton X-ray generation) project. 
Table 1: Proof-of-Principle experiment on laser pulse stacking.

\begin{tabular}{|l|c|c|}
\hline Items & Measured Values & Calculated Values \\
\hline \hline First experiment with $90 \%$ reflectivity & non & \\
\hline Length of Cavity & 0.61 & 1 \\
Transmission & 6 & $10 \pm 2$ \\
Enhancement Factor & $40 \pm 5$ & $30 \pm 6$ \\
Finesse & non & $0.90 \pm 0.02$ \\
Reflectivity & non & \\
\hline Final experiment with $99.99 \%$ reflectivity & non & $20940 \pm 1904$ \\
\hline Length of Cavity & non & $31414 \pm 2856$ \\
Transmission & non & $0.9999 \pm 0.00001$ \\
\hline Enhancement Factor & non & 1 \\
Finesse & & \\
\hline
\end{tabular}

